\title{
Surface oxidation of activated electrospun carbon nanofibers and their adsorption performance for benzene, butanone and ethanol
}

Yu Bai ${ }^{\mathrm{a}}$, Zheng-Hong Huang ${ }^{\mathrm{a},{ }^{*},}$ Feiyu Kang ${ }^{\mathrm{a}, \mathrm{b}, *}$

${ }^{a}$ Key Laboratory of Advanced Materials (MOE), School of Materials Science and Engineering, Tsinghua University, Beijing 100084, China

${ }^{\mathrm{b}}$ Graduate School at Shenzhen, Tsinghua University, Shenzhen 518055, China

${ }^{*}$ Corresponding author. Tel/Fax: +86 10 62773752. E-mail address: zhhuang@mail.tsinghua.edu.cn (Z.-H. Huang), fykang@tsinghua.edu.cn (F. Kang).

\begin{abstract}
Carbon nanofibers prepared by electrospinning and subsequent steam activation were oxidized by treatment in $\mathrm{HNO}_{3}$ and $\mathrm{HNO}_{3} / \mathrm{H}_{2} \mathrm{SO}_{4}$ mixture. Surface morphology and degree of graphitic structure of oxidized nanofibers are affected mildly by acid oxidation according to SEM and Raman measurement. Nitrogen adsorption/desorption isotherms demonstrate decrease in specific surface area and pore volume of carbon nanofibers after oxidation. XPS spectra exhibit that oxidized nanofibers possess higher surface oxygen content than pristine nanofibers. Higher water adsorption capacities on oxidized nanofibers determined by water adsorption isotherms suggest the improvement of surface polarity and hydrophilicity. Furthermore, adsorption isotherms of benzene, butanone and ethanol show that surface oxidation enhances the adsorption tendency for butanone and ethanol on nanofibers due to increased surface polarity, indicating that the adsorption selectivity to polar organic compounds is improved.
\end{abstract}

Key words: activated electrospun carbon nanofibers; surface oxidation; adsorption 


\section{Introduction}

Porous carbon nanofibers are excellent candidate for gas (inorganic and organic compounds) adsorption owing to their high specific surface area and developed narrow pore structure $[1,2]$. Recently, electrospinning has become a preferred method to prepare carbon nanofibers effectively and conveniently [3]. Activated carbon nanofibers prepared by electrospinning and following activation have received much attention. The freestanding monolithic characteristic and developed microporous structure can enhance the potential of activated electrospun carbon nanofibers (AECNFs) for practical adsorption application $[4,5]$.

Surface chemical properties have significant influences on the practical performance of carbonaceous materials as gas adsorbents [6,7]. Surface functionalization can change the reactivity and selectivity of carbon surface, which plays an important role on the adsorption for specific organic molecules. Introduction of polar functional groups can promote the polarity and wettability of carbonaceous materials and consequently benefit the adsorption performance, especially for polar organic molecules [8]. Surface functionalization of carbon nanotubes (CNTs) and activated carbons (ACs) has been studied in literature [6, 9-11]. Lu observed that NaOCl-modified CNTs had more hydrophilic surface, which improved benzene, toluene and ethylbenzene adsorption [6]. Kim reported that toluene, benzene and methanol adsorption quantities of ACs impregnated in phosphoric acid were enhanced [9]. However, little data about the effects of surface functionalization on the physicochemical properties and gas adsorption performance of porous carbon nanofibers, especially AECNFs, has been reported.

Chemical oxidation by acid treatment is the most widely used method for surface functionalization, which can improve the polarity and hydrophilicity of carbon surface $[12,13]$. 
Thus, in the present work, AECNFs were treated in oxidative acid to achieve surface oxidation. Effects of oxidation on the morphology, pore structure, surface chemical properties of AECNFs are analyzed. Furthermore, adsorption performances of pristine and oxidized carbon nanofibers for water, benzene, butanone and ethanol are determined by static adsorption isotherms. Benzene (non-polar), butanone and ethanol (polar) are selected with an aim to study the influence of surface oxidation on adsorption performance for organic compounds with different polarity.

\section{Experimental}

\subsection{Preparation of pristine AECNFs}

$10 \mathrm{wt} . \%$ polyacrylonitrile $(\mathrm{Mw}=150,000$, ONTARIO, New York, USA) was dissolved in N,N-dimethylformamide (Beijing Modern Eastern Finechemical Co., Ltd, China) and stirred at $65{ }^{\circ} \mathrm{C}$ for $12 \mathrm{~h}$ to obtain a homogeneous solution. The solution was electrospun into nanofibers, and electrospinning equipment were described in our previous work [14]. The applied voltage was $25 \mathrm{kV}$, the distance between the tip and the collector was $19 \mathrm{~cm}$, and the flow rate of solution was $1 \mathrm{~mL} \mathrm{~h}^{-1}$. The electrospun fibers were stabilized by heating to $280{ }^{\circ} \mathrm{C}$ at a rate of $1{ }^{\circ} \mathrm{C} \mathrm{min}^{-1}$ in air and holding for $2 \mathrm{~h}$. Then, the fibers (about $1.2 \mathrm{~g}$ ) were heated to $800{ }^{\circ} \mathrm{C}$ (heating rate: $5{ }^{\circ} \mathrm{C} \mathrm{min}^{-1}$ ) in nitrogen and activated by supplying 30 vol.\% water steam at $800{ }^{\circ} \mathrm{C}$ for $30 \mathrm{~min}\left(800{ }^{\circ} \mathrm{C}\right.$ is favorable for activation process according to our previous work [14]). The resultant carbon nanofiber was denoted as AECNF.

\subsection{Acid oxidation of carbon nanofibers}

$\mathrm{HNO}_{3}$ (68 wt.\%) and $\mathrm{H}_{2} \mathrm{SO}_{4}(95 \sim 98$ wt.\%) were purchased from Beijing Modern Eastern Finechemical Co., Ltd, China and used without further purification. Carbon nanofibers (90 mg) were impregnated into $\mathrm{HNO}_{3}(35 \mathrm{~mL})$, and the system was continuously stirred and maintained at 
room temperature for $24 \mathrm{~h}$. Then, the fibers were filtered and adequately washed with water until the $\mathrm{pH}$ of the filtrate was 7 . The resultant sample was dried under vacuum $\left(80^{\circ} \mathrm{C}\right)$ for $12 \mathrm{~h}$, and denoted as AECNF-N. Similarly, sample treated by $\mathrm{HNO}_{3} / \mathrm{H}_{2} \mathrm{SO}_{4}$ mixture (volume ratio = 1:3) for $12 \mathrm{~h}$ was denoted as AECNF-NS.

\subsection{Materials characterization}

Microscopic morphology and structure of fibers were examined by scanning electron microscope (SEM, LEO-1530, Germany) and Raman spectroscopy (Renishaw Invia RM200, England). Pore structural properties were determined from $\mathrm{N}_{2}$ adsorption/desorption at $77 \mathrm{~K}$ (Quantachrome Autosorb-1, USA). Various fibers were degassed at $195^{\circ} \mathrm{C}$ for about $18 \mathrm{~h}$ before the test. Surface functional groups and chemical concentration of samples were investigated by X-ray photoelectron spectroscopy (XPS, ESCALAB250Xi, ThermoFisher). Easydrop D04010 (KRUSS GmbH Germany) was used to measure the water contact angles of samples.

\subsection{Vapor adsorption measurement}

A static adsorption system (Belsorp-max, Japan) was used to obtain the water, benzene, butanone and ethanol adsorption isotherms of various samples at $20^{\circ} \mathrm{C}$. Nanofibers (about $30 \mathrm{mg}$ ) were degassed at $195{ }^{\circ} \mathrm{C}$ for $18 \mathrm{~h}$ under nitrogen flow. The organic liquids used to generate the vapors were fully degassed by repeated evacuation before the test. Saturation pressures of water, benzene, butanone and ethanol at $20{ }^{\circ} \mathrm{C}$ calculated by Antoine equation [15] are 2.34, 10.03, 9.46 and $5.87 \mathrm{kPa}$, respectively. The respective dipole moment of benzene, butanone and ethanol are $0.0,2.8$ and $1.7 \mathrm{D}[15]$.

\section{Results and discussion}

\subsection{Characterization of pristine and oxidized as-obtained nanofibers}


SEM images in Fig. 1 show that both pristine and oxidized nanofibers exhibit long fibrous morphology. It can be observed that treatment in acid does not have obvious effects on the surface and diameter of single nanofiber. The three samples possess almost the same fiber diameter, and their average diameters range from 175 to $200 \mathrm{~nm}$ approximately. However, for AECNF-NS, more short fibers or fiber fragments exist, which is in accord with the results reported in some studies about acid-treated carbon fibers or CNTs $[12,16]$.
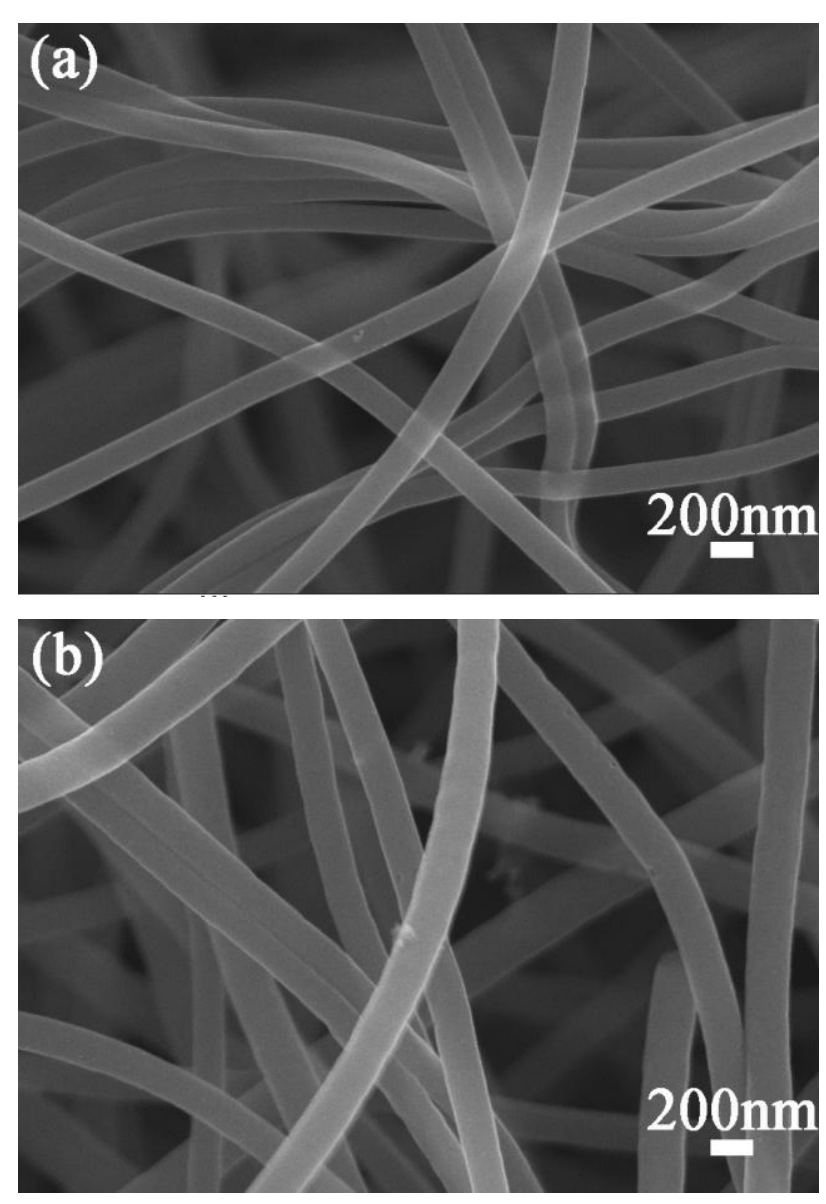


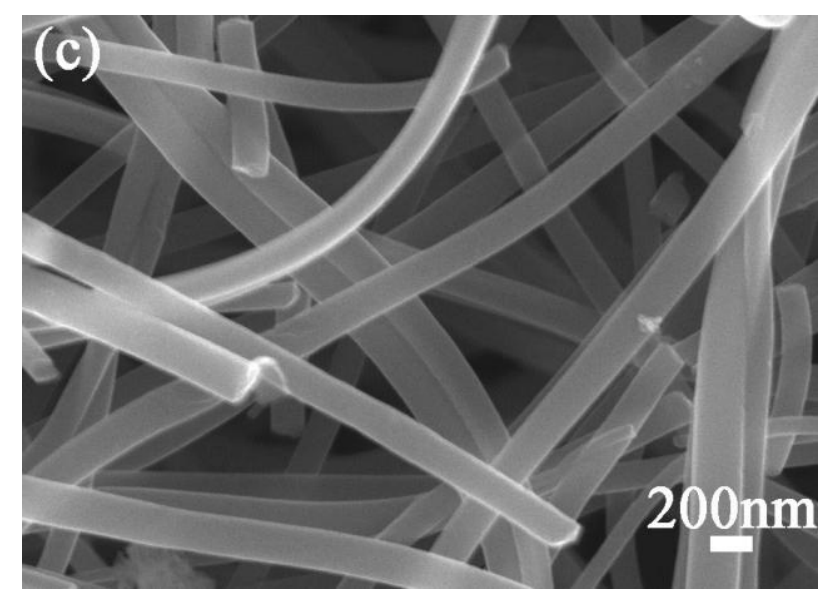

Fig. 1. SEM images of (a) AECNF, (b) AECNF-N and (c) AECNF-NS.

Raman spectra in Fig. S1 (See details in the Supporting Information) show that all the three samples display two broad overlapping peaks at 1355 (D peak, the defects and disordered band) and 1590 (G peak, the ordered graphitic band) $\mathrm{cm}^{-1}$, and treatment in acid does not obviously affect the shape of spectra and position of peaks. Furthermore, the ratios of the integrated intensity of D peak to G peak, denoted as $\mathrm{I}_{\mathrm{D}} / \mathrm{I}_{\mathrm{G}}$, for AECNF, AECNF-N and -NS are 3.28, 3.31 and 3.40, respectively. Since the value of $I_{D} / I_{G}$ is dependable on the degree of graphitic structure [17], the slight increase in $\mathrm{I}_{\mathrm{D}} / \mathrm{I}_{\mathrm{G}}$ from AECNF to AECNF-NS indicates that the degree of graphitization is reduced mildly with the enhanced acid functionalization.

Pore structural properties of carbon nanofibers are investigated by nitrogen adsorption/desorption measurement. Fig. 2a shows $\mathrm{N}_{2}$ adsorption/desorption isotherms of three samples. The nitrogen adsorption was nearly completed at a low relative pressure $\left(\mathrm{P} / \mathrm{P}_{0}<0.05\right)$, and it shows a plateau over a wide range of relative pressures. Thus, all the isotherms belong to Type I by IUPAC classification, indicating that the nanofibers are typically microporous [18]. Water steam activation is an important method to improve the porous properties of carbon nanofibers. Micropores are created on the surface of individual nanofiber in the activation process due to the 
reaction between carbon and water $[4,14]$.

Nitrogen adsorption capacities at different relative pressures decrease from AECNF to AECNF-NS, suggesting that treatment in acid weakens the microporous structure of carbon nanofibers. Pore structural parameters of various nanofibers are listed in Table 1. The specific surface area and pore volume, which mainly result from micropores, are diminished from AECNF to AECNF-NS. Pore size distributions calculated by non-local density function theory [19] shown in Fig. $2 \mathrm{~b}$ reveal that all the three nanofibers possess multimodal microporous structure. However, decreased microporosity is observed with acid-treated nanofibers mainly due to the dramatic decrease in micropore volume with widths below $1 \mathrm{~nm}$ (Fig. 2b). Similar change was also observed by Yu who studied the functionalization of carbon nanotube [20]. The above results suggest that some micropores on the surface of carbon nanofibers are destroyed by treatment in acid, especially $\mathrm{HNO}_{3} / \mathrm{H}_{2} \mathrm{SO}_{4}$ mixture.

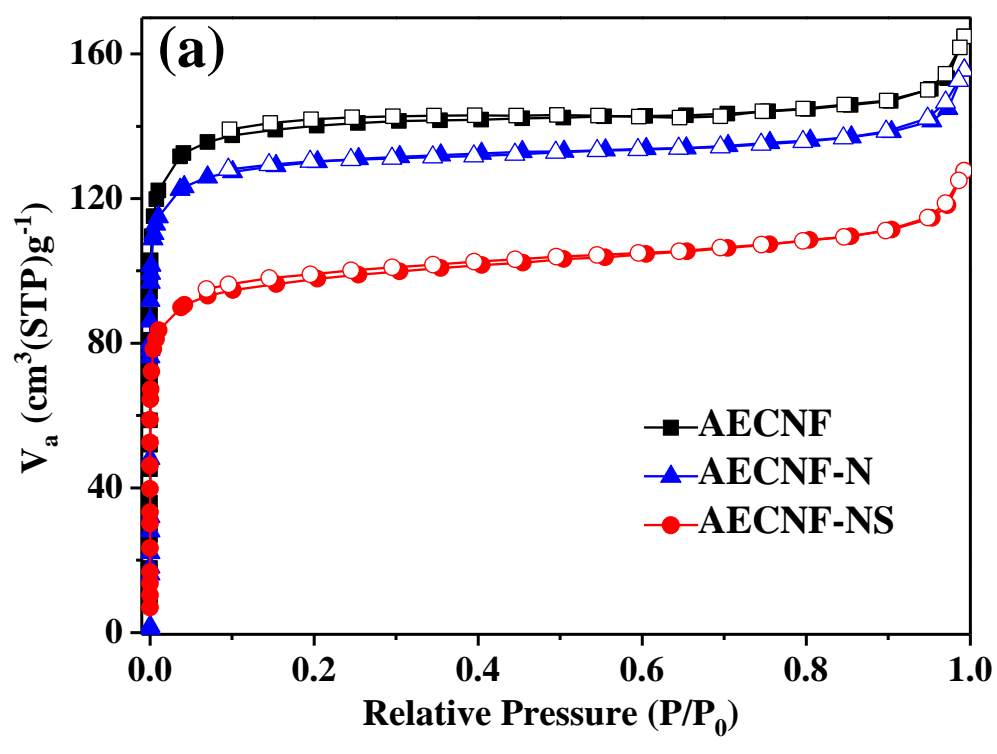




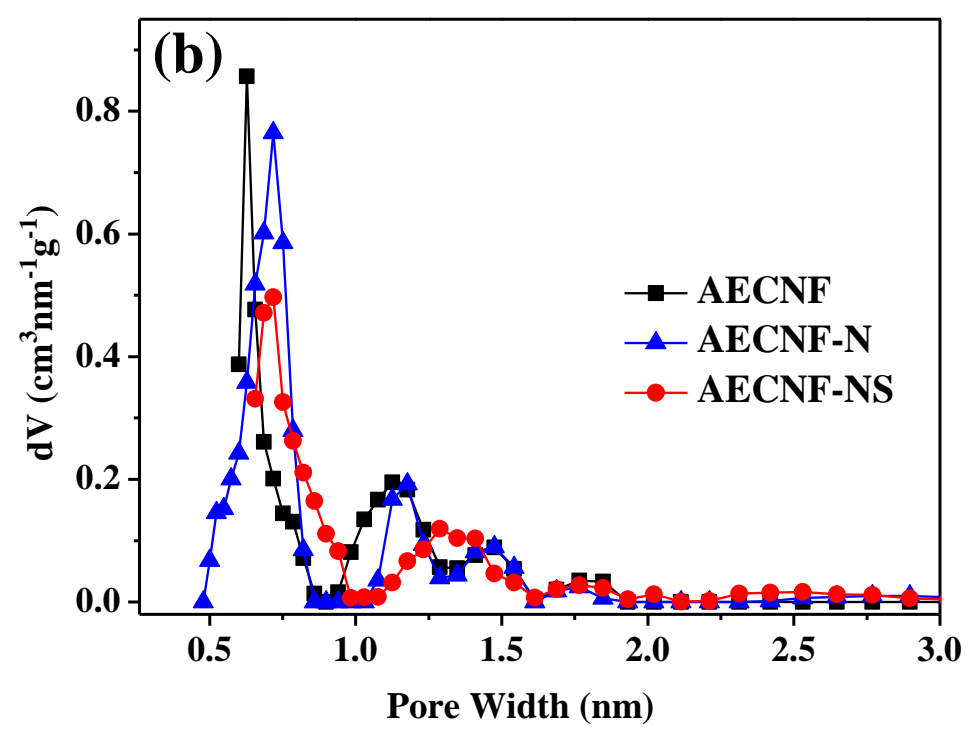

Fig. 2. (a) $\mathrm{N}_{2}$ adsorption/desorption isotherms at $77 \mathrm{~K}$ and (b) pore size distributions of pristine and acid-treated AECNFs.

Table 1 Pore structural parameters and surface chemistry composition of various nanofibers.

\begin{tabular}{llllllllll}
\hline Sample & $\begin{array}{l}\mathrm{S}_{\mathrm{T}}{ }^{\mathrm{a}} \\
\left(\mathrm{m}^{2} \mathrm{~g}^{-1}\right)\end{array}$ & $\begin{array}{l}\mathrm{S}_{\mathrm{m}}{ }^{\mathrm{b}} \\
\left(\mathrm{m}^{2} \mathrm{~g}^{-1}\right)\end{array}$ & $\begin{array}{l}\mathrm{V}_{\mathrm{T}}{ }^{\mathrm{C}} \\
\left(\mathrm{cm}^{3} \mathrm{~g}^{-1}\right)\end{array}$ & $\begin{array}{l}\mathrm{V}_{\mathrm{m}}{ }^{\mathrm{d}} \\
\left(\mathrm{cm}^{3} \mathrm{~g}^{-1}\right)\end{array}$ & $\begin{array}{l}\mathrm{C} \\
(\text { at.\% })\end{array}$ & $\begin{array}{l}\mathrm{O} \\
(\text { at.\% })\end{array}$ & $\begin{array}{l}\mathrm{C}-\mathrm{O} \\
(\text { at.\% })\end{array}$ & $\begin{array}{l}\mathrm{C}=\mathrm{O} \\
(\text { at.\% })\end{array}$ & $\begin{array}{l}\mathrm{O}=\mathrm{C}-\mathrm{O} \\
(\text { at.\% })\end{array}$ \\
\hline AECNF & 562 & 552 & 0.233 & 0.214 & 83.3 & 11.5 & 19.1 & 8.9 & 5.9 \\
AECNF-N & 520 & 509 & 0.220 & 0.199 & 79.8 & 13.7 & 19.0 & 7.4 & 8.4 \\
AECNF-NS & 383 & 361 & 0.178 & 0.146 & 76.7 & 17.3 & 18.0 & 8.7 & 9.9 \\
\hline
\end{tabular}

${ }^{\text {a }} \mathrm{S}_{\mathrm{T}}$ : specific surface area by $\alpha_{\mathrm{s}}$ method.

${ }^{\mathrm{b}} \mathrm{S}_{\mathrm{m}}$ : micropore surface area by t-plot method.

${ }^{\mathrm{c}} \mathrm{V}_{\mathrm{T}}$ : total pore volume according to the capacity of $\mathrm{N}_{2}$ adsorption isotherm at $\mathrm{P} / \mathrm{P}_{0}$ of 0.95 .

${ }^{\mathrm{d}} \mathrm{V}_{\mathrm{m}}$ : micropore volume by t-plot method.

XPS can provide important information on surface chemical composition and functional groups of various carbon nanofibers. Atomic ratios of carbon, oxygen obtained by XPS are summarized in Table 1. It can be observed that acid functionalization improves the surface oxygen content of 
carbon nanofibers, and AECNF-NS possesses the highest oxygen content, which confirms that treatment in acid introduces oxygen functional groups on the surface and achieves the surface oxidation of AECNFs. Fig. 3a-c show that XPS C1s spectrum can be deconvoluted into four peaks centered at about 284.7, 285.5, 287.1, $289.4 \mathrm{eV}$ which are assigned to $\mathrm{C}-\mathrm{C}$ (graphitic carbon), C-O (alcohol or ether carbon), $\mathrm{C}=\mathrm{O}$ (carbonyl) and $\mathrm{O}=\mathrm{C}-\mathrm{O}$ bonds (carboxyl or ester carbon), respectively [20]. Table 1 reveals that the overall content of $\mathrm{C}=\mathrm{O}$ and $\mathrm{O}=\mathrm{C}-\mathrm{O}$ groups on carbon surface increases with the degree of surface oxidation increasing. In addition, the peak at around $532 \mathrm{eV}$ for O1s spectra in Fig. 3d is corresponding to oxygen with a double bond to carbon, and the fact that this peak shifts to lower value after acid oxidation can be due to the higher presence of $\mathrm{C}=\mathrm{O}$ and $\mathrm{O}=\mathrm{C}-\mathrm{O}$ groups [21], which is in good agreement with the case of $\mathrm{C} 1 \mathrm{~s}$ spectra.

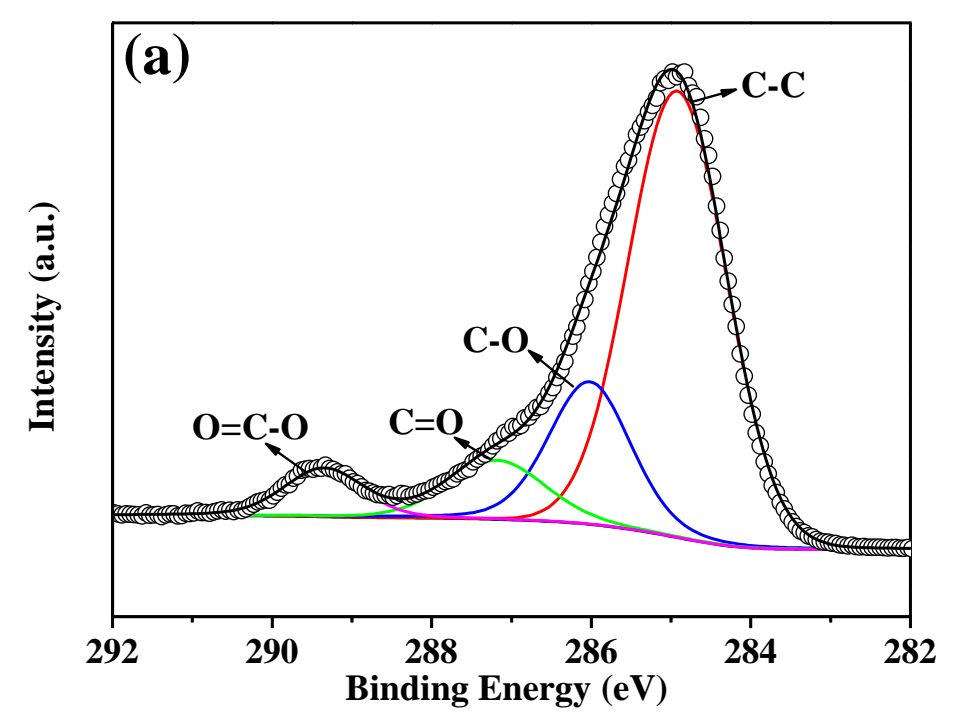



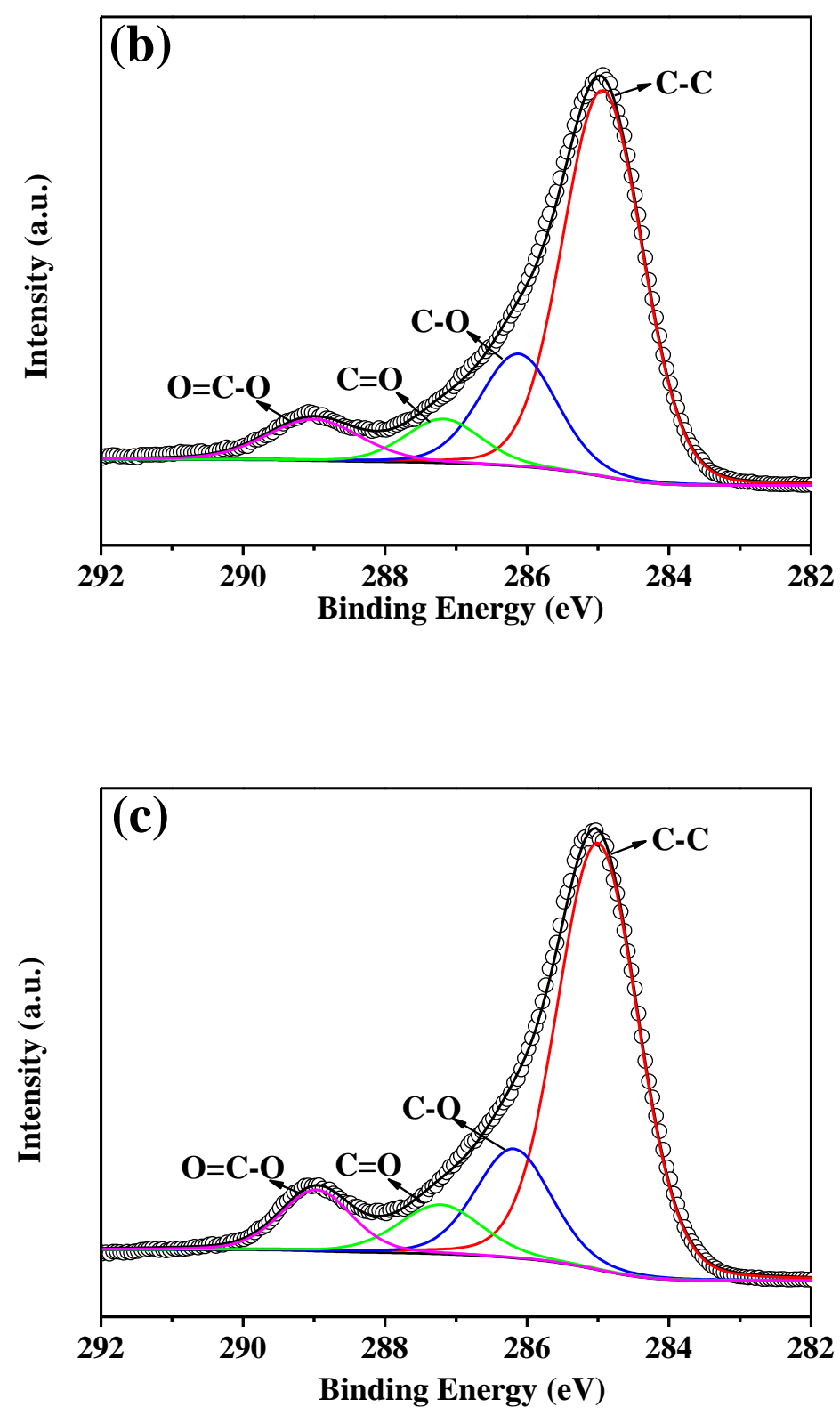


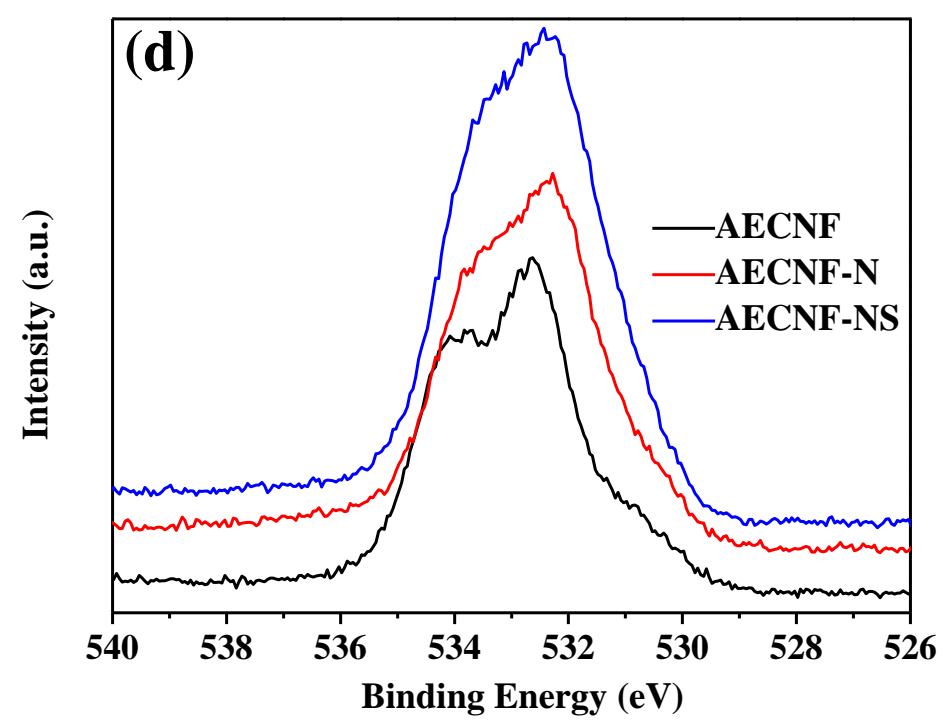

Fig. 3. XPS C1s high-resolution spectra of (a) AECNF, (b) AECNF-N and (c) AECNF-NS. (d)

O1s high-resolution spectra of three samples.

\subsection{Water adsorption performance of pristine and oxidized as-obtained nanofibers}

Water adsorption characteristics of various carbon nanofibers are evaluated by static water adsorption/desorption isotherms shown in Fig. 4. All the three isotherms go up gradually at low relative pressures. It is worth noting that water uptake quantities at low relative pressures $\left(\mathrm{P} / \mathrm{P}_{0}<0.35\right)$ are enhanced by surface oxidization, and AECNF-NS exhibits the highest adsorption capacities among the three samples. This fact suggests that the adsorption tendency of oxidized carbon nanofibers for water is enhanced, considering that adsorption quantities at low relative pressures can reflect the adsorption potential when micropores are not filled to the full [22]. Adsorption tendency for vapor is associated with porous structure and surface chemical properties of adsorbent materials [4]. The enhanced water adsorption on AECNF-N and -NS should be attributed to surface oxidation, rather than pore structural properties, since microporous structure 
is reduced observably after acid oxidation (Fig. 2 and Table 1). In addition, the water contact angle of AECNF is $31^{\circ}$, higher than the cases of AECNF-N $\left(22^{\circ}\right)$ and -NS $\left(19^{\circ}\right)$, demonstrating that oxidized carbon nanofibers have improved hydrophilicity. Surface oxidation improves the content of oxygen-containing groups constituting the polar sites [23], which leads to higher surface polarity and consequently more hydrophilic characteristic of AECNF-N and -NS compared to pristine AECNF. Water molecules can be adsorbed on the hydrophilic surface of electrospun nanofibers by polar interaction with functional groups [24]. Thus, AECNF-NS with the highest surface oxygen content possesses the strongest water adsorption tendency at low relative pressures.

However, water adsorption amounts of oxidized nanofibers become identical with those of pristine nanofibers at high relative pressures (Fig. 4), indicating that the decreased specific surface area and pore volume play more important roles on water adsorption performance of oxidized nanofibers than surface oxidation at high relative pressures.

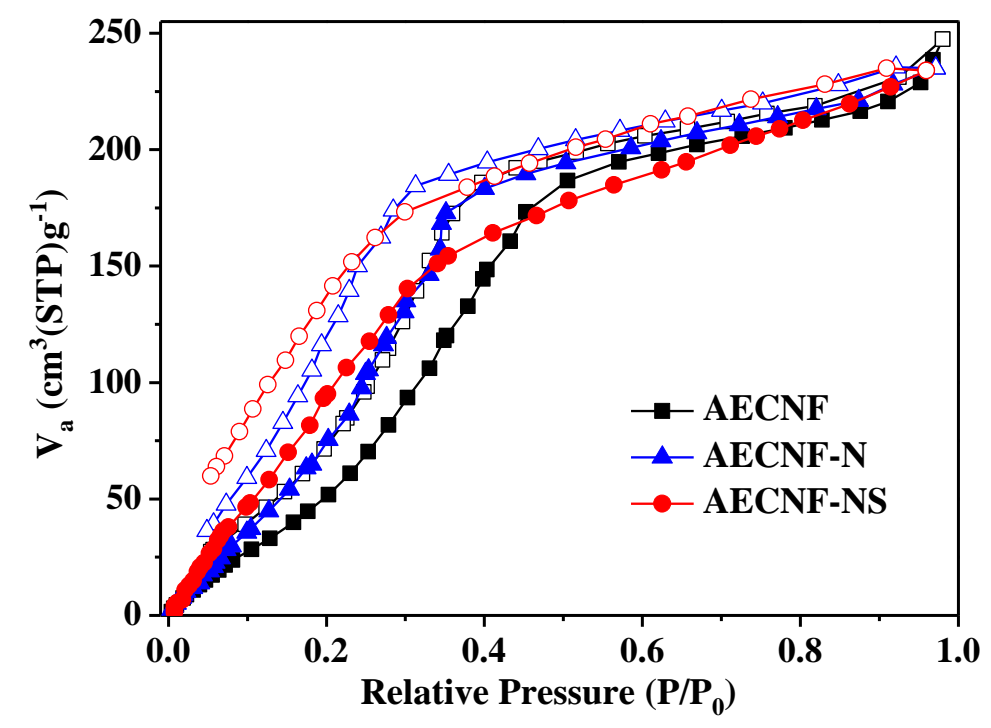

Fig. 4. Adsorption/desorption isotherms of water on three nanofibers at $20^{\circ} \mathrm{C}$. 
3.3. Benzene, butanone and ethanol adsorption performance of pristine and oxidized as-obtained nanofibers

Fig. 5 shows adsorption isotherms of benzene, butanone and ethanol on three nanofibers at $20{ }^{\circ} \mathrm{C}$. All isotherms exhibit a steep rise at extremely low relative pressures, suggesting strong attractive force between organic vapor and carbon surface [2]. For benzene, uptake quantities at different relative pressures reduce orderly from AECNF, AECNF-N to AECNF-NS. At P/P $\mathrm{P}_{0}$ of 0.9, the adsorbed benzene volume of AECNF, AECNF-N and -NS are 70, 61 and $51 \mathrm{~cm}^{3} \mathrm{~g}^{-1}$, respectively. For butanone, adsorption capacities of AECNF and AECNF-N are almost the same, while uptake quantities of AECNF-NS are lower than the cases of AECNF and AECNF-N. At P/P of 0.9 , the butanone adsorption quantities of AECNF, AECNF-N and -NS are 111, 112 and $87 \mathrm{~cm}^{3}$ $\mathrm{g}^{-1}$, respectively. For ethanol, uptake quantities of AECNF-NS are lower than the case of AECNF. At P/P0 of 0.9, the ethanol adsorption capacities of AECNF and -NS are 75 and $68 \mathrm{~cm}^{3} \mathrm{~g}^{-1}$, respectively. Porous structure of carbon adsorbents is a key point to dominate adsorption capacities for organic compounds [25]. The decrease in adsorbed benzene, butanone and ethanol volume on AECNF-NS is principally derived from the loss of specific surface area and pore volume. However, ethanol adsorption capacities of AECNF-N are higher than those of AECNF, which will be analyzed in the following part. 

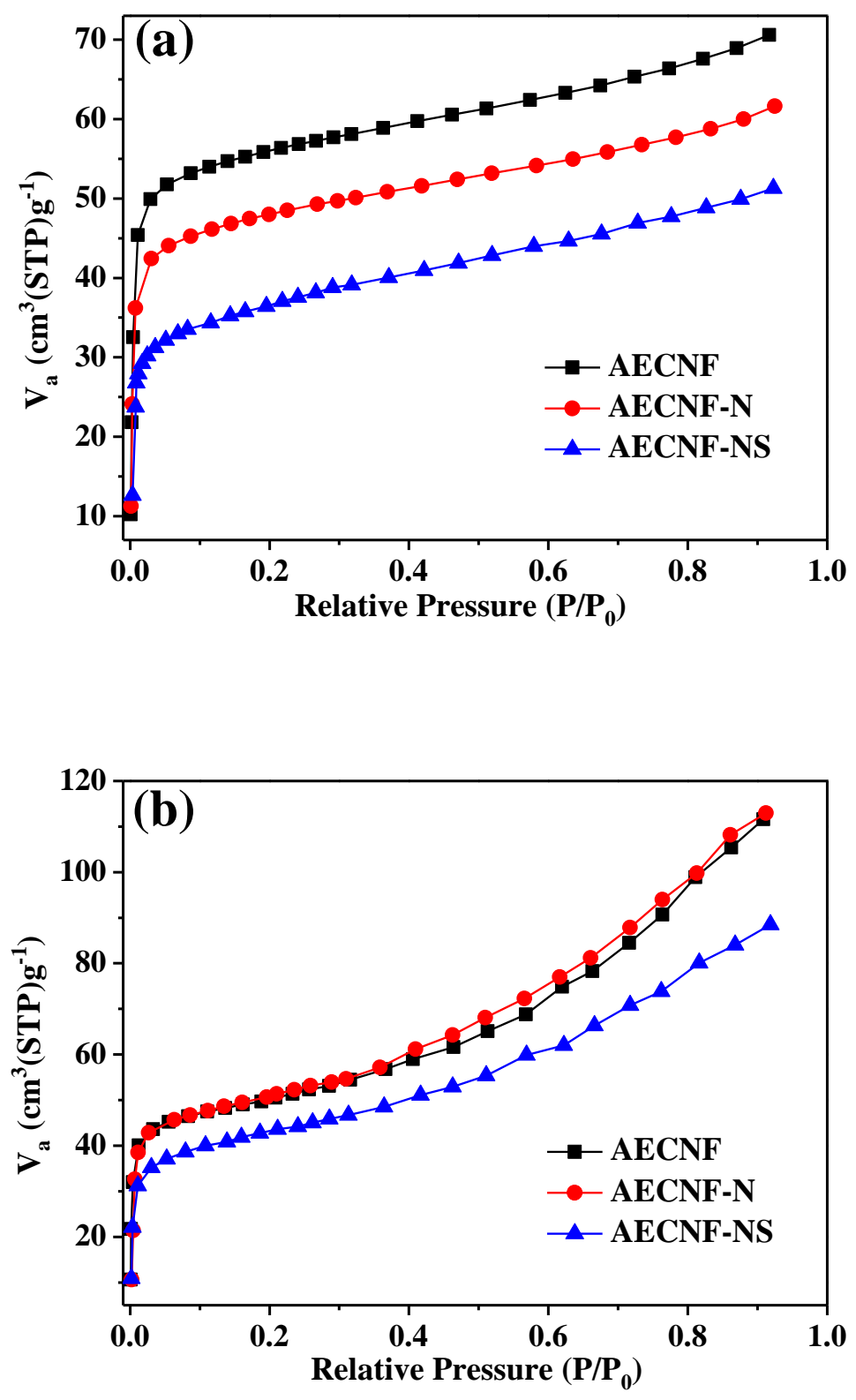


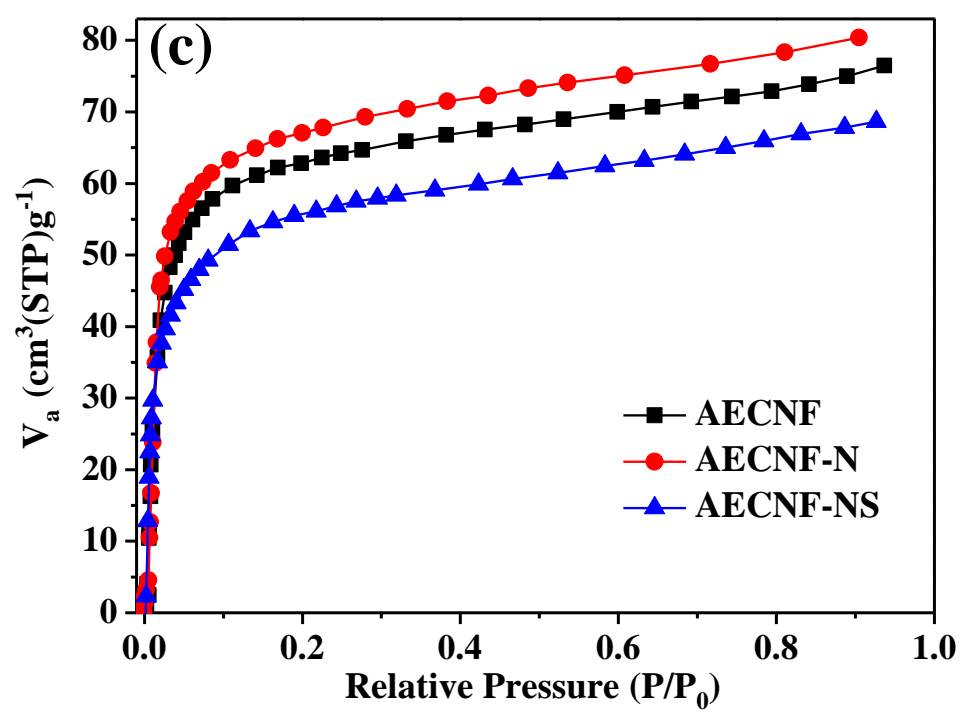

Fig. 5. Adsorption isotherms of (a) benzene (b) butanone and (c) ethanol on different nanofibers at $20{ }^{\circ} \mathrm{C}$

Furthermore, the enhancement of surface polarity of oxidized carbon nanofibers demonstrated by XPS and water adsorption isotherms can have different effects on benzene, butanone and ethanol adsorption, considering that benzene is a non-polar organic compound while butanone and ethanol are polar ones. Here, adsorption tendency for different organic compounds on AECNFs are studied for the first time. Adsorption capacity ratios of butanone and ethanol relative to benzene, $\mathrm{V}_{\mathrm{But}} / \mathrm{V}_{\text {Ben }}$ and $\mathrm{V}_{\mathrm{Eth}} / \mathrm{V}_{\text {Ben }}$, at different relative pressures, are obtained in Fig. 6, with an aim to intuitively highlight the influence of surface oxidation on benzene, butanone and ethanol adsorption. Similar processing was operated to determine the vapor adsorption selectivity of modified CNTs [26]. It can be observed that the ratios at most relative pressures $\left(\mathrm{P} / \mathrm{P}_{0}<0.7\right)$ increase with the degree of surface oxidation increasing, suggesting that oxidized carbon nanofibers exhibit higher adsorption selectivity for butanone and ethanol compared to pristine 
nanofibers. This fact can be attributed to the interaction between butanone or ethanol and polar surface on carbon nanofibers. Similar chemical behavior of the surface and the adsorbate can enhance the interaction between adsorbent and adsorbate [27]. Benzene, butanone and ethanol have very similar boiling point (around $80^{\circ} \mathrm{C}$ ), while butanone and ethanol exhibit higher polarity than benzene. Thus, the oxidized nanofibers with more polar surface related to higher oxygen content can allow for the enhancement of butanone and ethanol adsorption tendency compared to benzene. This can also explain why AECNF-N shows higher adsorption capacities for ethanol than AECNF (Fig. 5c). However, at high relative pressures, the values of $V_{\text {But }} / V_{\text {Ben }}$ for AECNF-NS are lower than those for AECNF-N, and the values of $\mathrm{V}_{\mathrm{Eth}} / \mathrm{V}_{\mathrm{Ben}}$ for AECNF-NS become identical with those for AECNF-N. Adsorption behavior at high relative pressures may be dominated by a complex mechanism. More oxygen-containing functional groups on the external surface of AECNF-NS may hinder the attachment of butanone and ethanol molecules to suitable adsorption sites, which leads to the decreased $\mathrm{V}_{\mathrm{But}} / \mathrm{V}_{\mathrm{Ben}}$ and $\mathrm{V}_{\mathrm{Eth}} / \mathrm{V}_{\mathrm{Ben}}$ at high relative pressures, even though surface oxidation benefits the polar organic compound uptake.

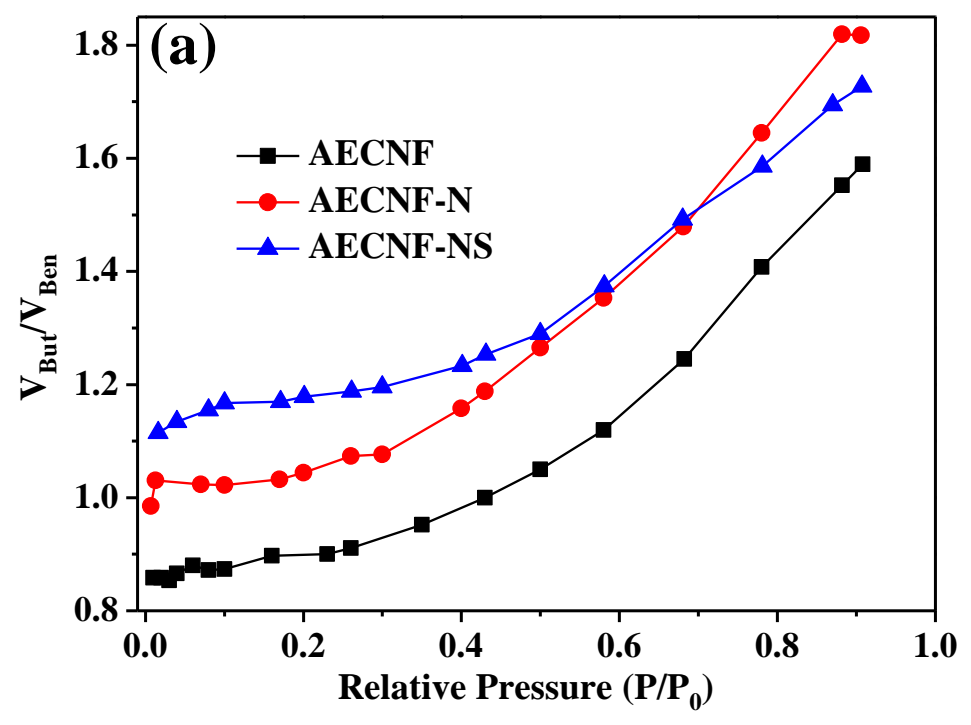




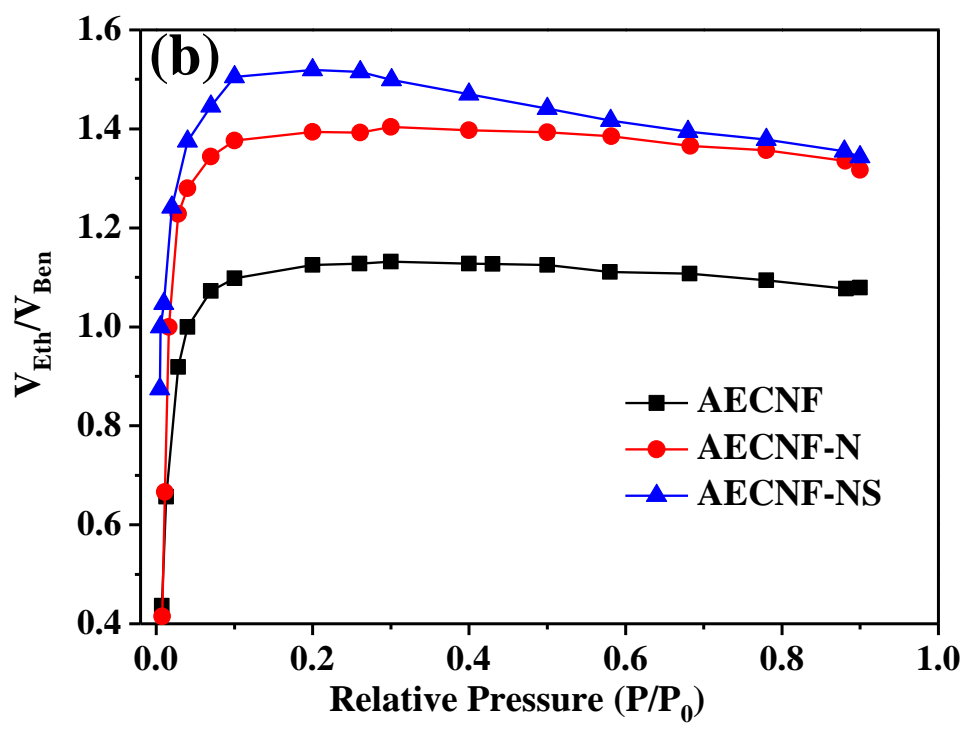

Fig. 6. Adsorption capacity ratio isotherms of (a) butanone and (b) ethanol relative to benzene on three samples at $20^{\circ} \mathrm{C}$.

\section{Conclusion}

Activated electrospun carbon nanofibers were oxidized by treatment in acid. Acid oxidation has adverse impacts on the morphology and degree of graphitic structure of carbon nanofibers. Content of oxygen functional groups on the surface of nanofibers increases with the degree of acid oxidation increasing. Improvement of surface polarity leads to enhanced water adsorption tendency on oxidized nanofibers in comparison to pristine ones, even though specific surface area and pore volume are reduced observably by acid oxidation. AECNF-NS exhibits the highest water adsorption capacities at low relative pressures. Furthermore, surface oxidation improves the adsorption tendency for butanone and ethanol relative to benzene on nanofibers, which results in increased values of $\mathrm{V}_{\mathrm{Bu}} / \mathrm{V}_{\mathrm{Ben}}$ and $\mathrm{V}_{\mathrm{Eth}} / \mathrm{V}_{\mathrm{Ben}}$ at most relative pressures from AECNF to AECNF-NS. 


\section{Acknowledgments}

The authors acknowledge the financial support of the cooperative project JST-MOST (No. 2011DFA50430), the National High Technology Research and Development Program of China

(863 Program-No. 2010AA064907) and the Program for New Century Excellent Talents in University (NCET-10-0496).

\section{References}

[1] M.R. Cuervo, E. Asedegbega-Nieto, E. Diaz, A. Vega, S. Ordonez, E. Castillejos-Lopez, I. Rodriguez-Ramos, Effect of carbon nanofiber functionalization on the adsorption properties of volatile organic compounds, J. Chromatogr. A 1188 (2008) 264-273.

[2] M.E. Ramos, P.R. Bonelli, A.L. Cukierman, M. Carrott, P.J.M. Carrott, Adsorption of volatile organic compounds onto activated carbon cloths derived from a novel regenerated cellulosic precursor, J. Hazard. Mater. 177 (2010) 175-182.

[3] A. Greiner, J.H. Wendorff, Electrospinning: A fascinating method for the preparation of ultrathin fibres, Angew. Chem.-Int. Edit. 46 (2007) 5670-5703.

[4] G.Y. Oh, Y.W. Ju, M.Y. Kim, H.R. Jung, H.J. Kim, W.J. Lee, Adsorption of toluene on carbon nanofibers prepared by electrospinning, Sci. Total Environ. 393 (2008) 341-347.

[5] K.J. Lee, N. Shiratori, G.H. Lee, J. Miyawaki, I. Mochida, S.-H. Yoon, J. Jang, Activated carbon nanofiber produced from electrospun polyacrylonitrile nanofiber as a highly efficient formaldehyde adsorbent, Carbon 48 (2010) 4248-4255.

[6] C. Lu, F. Su, S. Hu, Surface modification of carbon nanotubes for enhancing BTEX adsorption from aqueous solutions, Appl. Surf. Sci. 254 (2008) 7035-7041.

[7] A.A.M. Daifullah, B.S. Girgis, Impact of surface characteristics of activated carbon on 
adsorption of BTEX, Colloid Surf. A-Physicochem. Eng. Asp. 214 (2003) 181-193.

[8] Y.-J. Su, T.-H. Ko, J.-H. Lin, Preparation of ultra-thin PAN-based activated carbon fibers with physical activation, J. Appl. Polym. Sci. 108 (2008) 3610-3617.

[9] K.J. Kim, C.S. Kang, Y.J. You, M.C. Chung, M.W. Woo, W.J. Jeong, N.C. Park, H.G. Ahn, Adsorption-desorption characteristics of VOCs over impregnated activated carbons, Catal. Today 111 (2006) 223-228.

[10] H.H. Cho, B.A. Smith, J.D. Wnuk, D.H. Fairbrother, W.P. Ball, Influence of surface oxides on the adsorption of naphthalene onto multiwalled carbon nanotubes, Environ. Sci. Technol. 42 (2008) 2899-2905.

[11] F.S. Su, C.Y. Lu, S.K. Hu, Adsorption of benzene, toluene, ethylbenzene and p-xylene by NaOCl-oxidized carbon nanotubes, Colloid Surf. A-Physicochem. Eng. Asp. 353 (2010) 83-91.

[12] M.L. Toebes, E.M.P. van Heeswijk, J.H. Bitter, A.J. van Dillen, K.P. de Jong, The influence of oxidation on the texture and the number of oxygen-containing surface groups of carbon nanofibers, Carbon 42 (2004) 307-315.

[13] H.S. Oh, K. Kim, Y.J. Ko, H. Kim, Effect of chemical oxidation of CNFs on the electrochemical carbon corrosion in polymer electrolyte membrane fuel cells, Int. J. Hydrogen Energy 35 (2010) 701-708.

[14] M.-X. Wang, Z.-H. Huang, T. Shimohara, F. Kang, K. Liang, NO removal by electrospun porous carbon nanofibers at room temperature, Chem. Eng. J. 170 (2011) 505-511.

[15] I.M. Smallwood, Handbook of arganic solvent properties, Halsted Press, New York, 1996.

[16] Y.H. Li, S.G. Wang, Z.K. Luan, J. Ding, C.L. Xu, D.H. Wu, Adsorption of cadmium(II) from 
aqueous solution by surface oxidized carbon nanotubes, Carbon 41 (2003) 1057-1062.

[17] A.B. Garcia, I. Camean, I. Suelves, J.L. Pinilla, M.J. Lazaro, J.M. Palacios, R. Moliner, The graphitization of carbon nanofibers produced by the catalytic decomposition of natural gas, Carbon 47 (2009) 2563-2570.

[18] S. Brunauer, L.S. Deming, W.E. Deming, E. Teller, On a theory of the van der Waals adsorption of gases, J. Am. Chem. Soc. 62 (1940) 1723-1732.

[19] C. Lastoskie, K.E. Gubbins, N. Quirke, Pore-size distribution analysis of microporous carbons - a density-functional theory approach, J. Phys. Chem. 97 (1993) 4786-4796.

[20] F. Yu, J. Ma, Y.Q. Wu, Adsorption of toluene, ethylbenzene and m-xylene on multi-walled carbon nanotubes with different oxygen contents from aqueous solutions, J. Hazard. Mater. 192 (2011) 1370-1379.

[21] D. Pantea, H. Darmstadt, S. Kaliaguine, L. Summchen, C. Roy, Electrical conductivity of thermal carbon blacks - Influence of surface chemistry, Carbon 39 (2001) 1147-1158.

[22] C.T. Hsieh, W.Y. Chen, Gaseous adsorption of carbon tetrachloride onto carbon nanofiber arrays prepared by template-assisted synthesis, Diamond Relat. Mater. 16 (2007) 1945-1949.

[23] R.H. Bradley, B. Rand, On the physical adsorption of vapors by microporous carbons, J. Colloid Interface Sci. 169 (1995) 168-176.

[24] M.M. Dubinin, V.V. Serpinsky, Isotherm equation for water vapor adsorption by microporous carbonaceous adsorbents, Carbon 19 (1981) 402-403.

[25] J.-H. Tsai, H.-M. Chiang, G.-Y. Huang, H.-L. Chiang, Adsorption characteristics of acetone, chloroform and acetonitrile on sludge-derived adsorbent, commercial granular activated carbon and activated carbon fibers, J. Hazard. Mater. 15 (2008) 1183-1191. 
[26] E.C. Vermisoglou, V. Georgakilas, E. Kouvelos, G. Pilatos, K. Viras, G. Romanos, N.K. Kanellopoulos, Sorption properties of modified single-walled carbon nanotubes, Microporous Mesoporous Mat. 99 (2007) 98-105.

[27] T. Garcia, R. Murillo, D. Cazorla-Amoros, A.M. Mastral, A. Linares-Solano, Role of the activated carbon surface chemistry in the adsorption of phenanthrene, Carbon 42 (2004) 1683-1689. 


\section{Graphical Abstract}

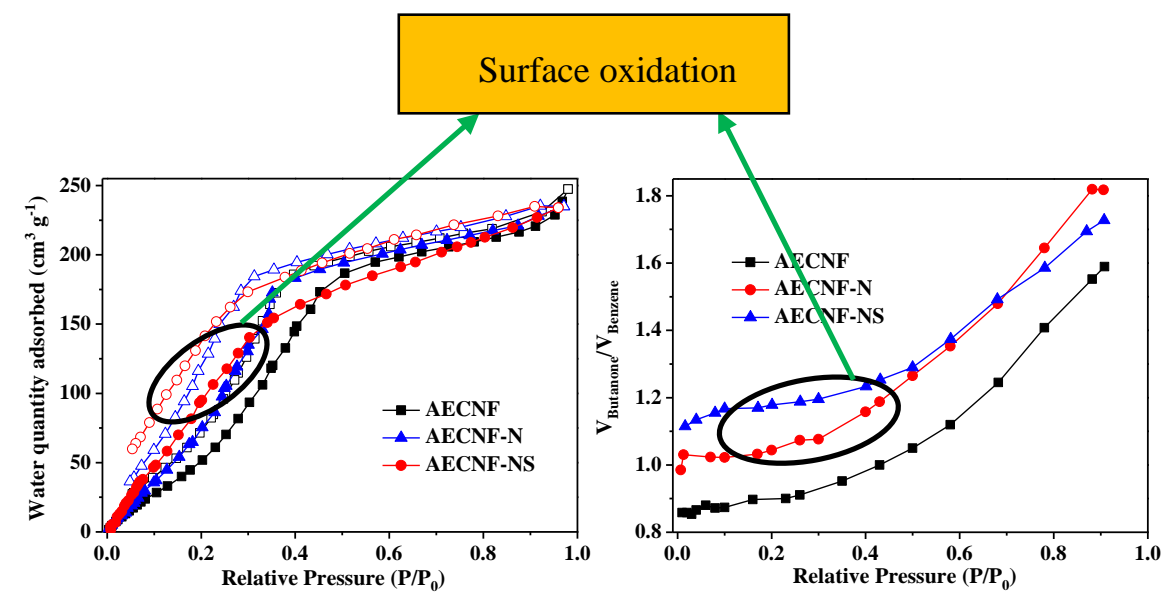

Surface oxidation of activated electrospun carbon nanofibers by treatment in acid enhanced the adsorption tendency for butanone relative to benzene, suggesting that the oxidized carbon nanofibers can be used as effective adsorbents for polar organic compounds. 